\title{
Percepção dos profissionais de saúde em relação à implantação do Modelo de Atenção às Condições Crônicas
}

\author{
Perception of the health carries in relation to Chronic Care Model
}

Laís Carolini Theis ${ }^{1}$

Orcid: https://orcid.org/0000-0001-6346-8661 Saulo Vinicíus Rosa ${ }^{3}$

Orcid: https://orcid.org/0000-0002-7107-9575

\author{
Dhaniel Marinho Mikosz ${ }^{2}$ \\ Orcid: https://orcid.org/0000-0002-5241-8324 \\ Simone Tetu Moysés ${ }^{4}$ \\ Orcid: https://orcid.org/0000-0003-4861-9980
}

Thyago Proença de Moraes 5

Orcid: https://orcid.org/0000-0002-2983-3968

\begin{abstract}
Resumo
Introdução: as condições crônicas não transmissíveis representam a maior causa de morbimortalidade no Brasil. O Modelo de Atenção às Condições Crônicas foi implantado no estado do Paraná como estratégia de enfrentamento ao cuidado crônico. Objetivo: avaliar a implantação do Modelo de Atenção às Condições Crônicas por meio da percepção dos profissionais de saúde. Materiais e Métodos: estudo exploratório, descritivo, com abordagem qualitativa, realizado em um serviço de atenção primária à saúde e um serviço de atenção ambulatorial especializada em uma região de saúde do estado do Paraná. Participaram do estudo sete profissionais da atenção especializada e onze profissionais da atenção primária. Os dados foram coletados por meio de seis encontros de grupos focais, cujas discussões foram norteadas pelo instrumento de avaliação do modelo de atenção às condições crônicas (IEMAC ARCHO 36), sendo submetidos à análise de conteúdo temática proposta por Bardin. Resultados: os relatos dos profissionais permitiram analisar a estratégia de implantação de acordo com as seis dimensões propostas pelo modelo: organização do sistema de saúde; saúde compartilhada; modelo assistencial; autocuidado; apoio à tomada de decisão; e sistemas de informação. Identificou-se que a implantação do modelo apresenta fragilidades quanto aos indicadores de saúde, mapa de ações interprofissionais, plano de autocuidado apoiado e sistemas de informação. E, também, avanços relacionados à organização da rede de atenção, estratificação de risco e desmedicalização de idosos. Conclusão: para atingir a efetividade do Modelo de Atenção às Condições Crônicas, faz-se necessário o completo desenvolvimento de suas dimensões para a garantia da qualidade e a melhoria do cuidado crônico.
\end{abstract}

Palavras-chave: doença crônica; modelos de assistência à saúde; Sistema único de saúde

\section{Abstract}

Introduction: chronic non-communicable conditions represent the major cause of morbidity and mortality in the country. The Chronic Care Model was implemented in the state of Paraná as a strategy to face chronic care. Objective: to evaluate the implementation of the Chronic Care Model through the perception of health professionals. Materials and

\footnotetext{
${ }^{1}$ Pontifícia Universidade Católica do Paraná. E-mail: laistheis@gmail.com

2 Prefeitura Municipal de Balsa Nova. E-mail: dhaniel.marinho@gmail.com

${ }^{3}$ Pontifícia Universidade Católica do Paraná. E-mail: sauloviinicius@hotmail.com

${ }^{4}$ Pontifícia Universidade Católica do Paraná . E-mail: simone.moyses@pucpr.br

${ }^{5}$ Pontifícia Universidade Católica do Paraná. E-mail: thyago.moraes@pucpr.br
} 
Methods: exploratory, descriptive study with a qualitative approach, carried out in a primary health care service and an outpatient specialized care service in a health region in the state of Paraná. Seven professionals from specialized care and eleven professionals from primary health care participated in the study. Data were collected through six focus group meetings, whose discussions were guided by the validated instrument for assessing the model of care for chronic conditions (IEMAC ARCHO 36), and were subjected to thematic content analysis proposed by Bardin. Results: the professionals' reports made it possible to analyze the implementation strategy according to the six dimensions proposed by the model: organization of the health system; shared health; care model; self-care; support for decision making; and information systems. It was identified that the implementation of the model has weaknesses in terms of health indicators, map of interprofessional actions, supported self-care plan, and information systems. And advances, related to the organization of the care network, risk stratification and medicalization of the elderly. Conclusion: to achieve the effectiveness of the Chronic Care Model, it is necessary to fully develop its dimensions to guarantee quality and improve chronic care.

Keywords: Chronic disease. Healthcare models. Unified health system.

\section{Introdução}

As doenças crônicas não transmissíveis (DCNT) são condições crônicas complexas e multifatoriais, caracterizadas por início gradual e progressivo, com prognóstico usualmente indefinido e incurável. Devido ao longo curso da doença, o quadro clínico pode oscilar ao longo do tempo, apresentando períodos de agudização, incapacidades e morte precoce ${ }^{1}$.

A maior carga de DCNT é representada pelas doenças cardiovasculares, respiratórias crônicas, câncer e diabetes. A Organização Mundial de Saúde atribui essa carga aos efeitos negativos da globalização e urbanização acelerada, bem como aos principais fatores de risco associados, como: consumo abusivo de bebidas alcoólicas; tabagismo; comportamento sedentário; e alimentação com alto teor calórico. As DCNT constituem a maior carga de morbimortalidade no mundo, responsáveis por $70 \%$ das mortes globais ${ }^{2}$.

Essa situação atinge indivíduos de todas as camadas socioeconômicas. Entretanto, as mortes por DCNT afetam predominantemente os países em desenvolvimento, nos quais cerca de um terço dos óbitos ocorrem em pessoas com menos de 60 anos de idade, enquanto nos países desenvolvidos a mortalidade prematura (faixa etária de 30 a 69 anos) corresponde a menos de $13 \%$ dos $\operatorname{casos}^{3}$. No Brasil, as DCNT corresponderam a $75,8 \%$ das mortes no ano de $2015^{4}$. Estudos mostram que cerca de $45 \%$ da população adulta brasileira refere ter pelo menos uma DCNT, o equivalente a 54 milhões de pessoas ${ }^{5}$.

Além do elevado número de mortes prematuras, as doenças crônicas não transmissíveis acarretam a perda da qualidade de vida, devido ao alto grau de limitação e incapacidade para as atividades de vida diária e aos impactos econômicos para famílias, comunidades e a sociedade em geral ${ }^{3}$. Desse modo, representam um desafio para muitos países no mundo, por estar crescendo de forma alarmante e, ainda, por defrontar a capacidade dos sistemas de saúde em suprir as demandas geradas por situações persistentes, que exigem certo nível de cuidado permanente ${ }^{3,6}$.

Devido à magnitude e necessidade de atenção às pessoas com doenças crônicas, a Organização das Nações Unidas lançou em 2012 o plano global de ações para prevenção e controle das $\mathrm{DCNT}^{7}$. O Brasil participou ativamente da ação global e lançou o Plano de Ações Estratégicas para enfrentamento das DCNT no Brasil (2011-2022), que pactuou metas e indicadores para redução dos fatores de riscos e complicações das $\mathrm{DCNT}^{8}$.

Mendes propôs como estratégia de enfrentamento a implantação do Modelo de 
Atenção às Condições Crônicas (MACC) ${ }^{9}$, desenvolvido para o âmbito do Sistema Único de Saúde (SUS) no Brasil, baseado nos conceitos de três modelos: o Modelo de Atenção Crônica ${ }^{10}$, o Modelo da Determinação Social de Saúde ${ }^{11}$ e o Modelo da Pirâmide de Risco ${ }^{12}$.

O Modelo de Atenção Crônica foi desenvolvido nos Estados Unidos, na década de 1990. Ele prevê resultados clínicos funcionais por meio de interações produtivas entre pessoas usuárias ativas e informadas e equipe de saúde proativa e preparada. De modo que ambos tenham acesso à recursos comunitários e um sistema de atenção à saúde organizado, com enfoque no autocuidado apoiado, com suporte gerencial para tomada de decisões, com um sistema de informação clínica e um desenho do sistema de prestação de serviços ${ }^{10}$.

O Modelo da Determinação Social da Saúde foi proposto na década de 1990 por Dahlgreen e Whitehead e inclui os determinantes sociais da saúde em diferentes camadas concêntricas, de acordo com o nível de abrangência, desde uma camada mais proximal até uma camada mais distal. Enfatiza interações de estilo de vida individual, redes social e comunitária, condições de vida e trabalho e condições ambientais, culturais e socioeconômicas ${ }^{11}$.

O Modelo da Pirâmide de Risco foi desenvolvido e aplicado por uma operadora de planos de saúde dos Estados Unidos e se sustenta fortemente na estratificação dos riscos da população. Isso, por sua vez, define as estratégias de intervenção em autocuidado e no cuidado profissional por meio das tecnologias de gestão da clínica, da condição de saúde e da gestão de caso ${ }^{12}$.

As condições crônicas ultrapassam a definição das doenças crônicas, como diabetes, doenças cardiovasculares e canceres. Elas incluem todas as condições de saúde caracterizadas como enfermidades em que há sofrimento, mas não doenças que se possam inscrever nos padrões biomédicos das classificações internacionais de doenças, podendo citar as condições ligadas à manutenção da saúde por ciclo de vidas, como a puericultura ${ }^{9}$.

O Modelo de Atenção às Condições Crônicas é composto por cinco níveis que expressam espaços de determinação social de respostas sociais diferenciadas e convoca novas abordagens tecnológicas que sejam efetivas no processo de mudança de comportamento, tanto para o indivíduo, quanto para os profissionais e gestores em saúde. $\mathrm{O}$ nível 1 é aplicado à toda população e prevê intervenções de promoção à saúde como foco nos determinantes sociais intermediários, por meio de ações intersetoriais que visem melhoria habitacional, geração de emprego e renda, acesso ao saneamento básico, melhoria educacional e na infraestrutura publica 9 .

O nível 2 aplica-se a subgrupos com fatores de risco e prevê intervenções de prevenção das condições de saúde com enfoque nos determinantes proximais da saúde, ligados ao comportamento e ao estilo de vida, por meio de ações voltadas ao tabagismo, alimentação inadequada, inatividade física, excesso de peso e uso excessivo de álcool. O nível 3 é destinado à ações de gestão da condição de saúde para indivíduos com condição crônica simples, com intervenções sobre os fatores de risco biopsicológicos, como idade, gênero, hereditariedade, hipertensão arterial, diabetes mellitus, dislipidemia e depressão ${ }^{9}$.

O nível 4 engloba a gestão da condição de saúde para indivíduos com condição crônica complexa. Nesse nível são recomendadas ações de autocuidado, apoio e cuidado profissional equilibradamente e, devido à complexidade da condição crônica, recomenda-se que o indivíduo seja assistido pela equipe de Atenção Primária à Saúde (APS) e serviço especializado. O nível 5 prevê ações de gestão de caso para subpopulações com condição crônica muito complexa. A gestão de caso configura-se pela presença de um profissional de saúde que coordene 
a atenção à saúde que será prestada ao indivíduo nos diferentes pontos da rede de atenção à saúde e nos sistemas de apoio. Nesse nível, há forte influência do cuidado profissional e a presença do gestor de caso se faz necessária ${ }^{9}$.

O MACC tem sido implantado em várias regiões de saúde por meio do projeto Planificação da Atenção à Saúde (PAS), coordenado pelo Conselho Nacional dos Secretários de Saúde (CONASS) ${ }^{13}$, que incentiva a criação de Laboratórios de Inovação sobre Condições Crônicas (LIACC) na APS no marco das Redes de Atenção à Saúde, objetivando alcançar soluções para o problema universal da hegemonia das condições crônicas. Um dos produtos desses laboratórios seria a produção de evidência científica sobre o MACC no contexto de redes de atenção.

Os LIACC atuam como campos de experimentação e disseminação de inovações para o SUS. Nesse cenário, o estado do Paraná, por meio da Secretaria de Estado da Saúde do Paraná (SESA), implantou, no ano de 2014, o Modelo de Atenção às Condições Crônicas em uma região de saúde do estado ${ }^{14}$. A região foi escolhida como projeto piloto devido a disponibilidade dos gestores locais para mudanças no modelo de atenção à saúde. A implantação iniciou em uma unidade de Atenção Primária à Saúde (APS), em um município de pequeno porte e na Atenção Ambulatorial Especializada (AAE), em um município referência que atende todos os 30 municípios que compõe a região de saúde. A implantação do MACC iniciou em 2014 com ações de reorganização no modelo de atenção, estratificação de risco dos pacientes, organização de fluxo de atendimento e, no ano de 2017, ocorreu o LIACC.

Devido à implantação piloto, o governo estadual visa conhecer os resultados dessa experiência para orientar a melhor tomada de decisão dos gestores, aprimoramento da qualidade da gestão em saúde e julgamento do sucesso da política pública implementada. E, desse modo, definir se o modelo será implantado em todo o estado. Do mesmo modo, os gestores da PAS, para conhecer os sucessos e insucessos para planificação nacional. Dessa forma, este estudo objetiva avaliar a implantação do Modelo de Atenção às Condições Crônicas por meio da percepção dos profissionais de saúde.

\section{Materiais e Métodos}

\section{Amostra e tipo de estudo}

Estudo exploratório, descritivo, com abordagem qualitativa, realizado em dois municípios de uma região de saúde no noroeste do estado do Paraná, onde o MACC foi implantado. Em um município, a coleta de dados ocorreu em uma unidade de APS com Programa Estratégia de Saúde da Família. E no outro município, na AAE, que consiste em um centro de referência de especialidades para todos os 30 municípios da região de saúde. Justifica-se a escolha de ambos os serviços para o estudo pelo fato de serem os serviços pioneiros na implantação do modelo que ocorreu no ano de 2014.

Participaram do estudo sete profissionais do serviço de atenção ambulatorial especializada e onze profissionais do serviço de atenção primária à saúde, entre eles as categorias de médico, enfermeiro, agente comunitário de saúde, dentista, auxiliar de saúde bucal e de enfermagem.

Inicialmente foi realizado contato com os gestores de cada unidade para definir a data e o horário para o grupo focal. Os gestores da unidade foram responsáveis por listar os profissionais aptos a participar do estudo, de acordo com os critérios de inclusão da pesquisa. Não houve recusas para participar do estudo.

\section{Delineamento da pesquisa}

A coleta de dados foi realizada por meio de grupos focais e as discussões foram norteadas pelo instrumento Avaliação de Modelos de Atenção às Condições Crônicas (IEMAC-ARCHO36), 
um questionário para a autoavaliação de organizações sanitárias e sociais em relação a seu grau de implementação de um modelo de excelência na atenção a pessoas com doenças crônicas ${ }^{15}$, na versão traduzida e validada para o idioma português brasileiro ${ }^{16}$. O instrumento é idealizado para ser aplicado para equipes multidisciplinares em saúde por meio de grupos focais, $\mathrm{o}$ que proporciona discussões e reflexões acerca do modelo. É composto por seis dimensões: organização do sistema de saúde; saúde compartilhada; modelo assistencial; autocuidado; apoio na tomada de decisão clínica; e sistemas de informação ${ }^{15}$.

Os dados foram coletados no período de agosto a novembro de 2018 , no local de trabalho das equipes. Antes de iniciar as reuniões, os participantes se apresentaram e, em seguida, um dos pesquisadores apresentou a problemática, os objetivos e os participantes do estudo assinaram o Termo de Consentimento Livre e Esclarecido.

$\begin{array}{ccr}\text { No serviço de Atenção } & \end{array}$ realizados dois encontros de grupo focal com a equipe multiprofissional. $\mathrm{Na}$ Atenção Primária à Saúde foram realizados quatro encontros de grupo focal. A diferença do número de reuniões entre equipes se deu pelo período de discussão e reflexão que cada equipe julgou necessário acerca de cada uma das perguntas do instrumento IEMAC-ARCHO 36. Ao iniciar a reunião do grupo focal, os pesquisadores apresentaram o instrumento IEMAC-ARCHO 36 e orientaram a equipe sobre o correto preenchimento do mesmo, de modo que a equipe precisava debater cada um dos tópicos do instrumento $\mathrm{e}$ atribuir um valor ao tópico em consenso, os quais variavam entre zero e cem pontos. Sendo zero o item não implantado e/ou desenvolvido e cem o item plenamente implantado e/ou desenvolvido. Os pesquisadores gravaram as discussões e a análise de dados se deu por meio das falas e reflexões da equipe sobre cada dimensão do modelo.

\section{Critérios de Inclusão e Exclusão}

Constituíram-se critérios de inclusão: ser profissional de saúde do serviço de Atenção Primária à Saúde e/ou do serviço de Atenção Ambulatorial Especializada onde o MACC foi implantado; participar de ações rotineiras que envolvem o modelo; e ter pelo menos quatro anos de atuação no serviço. Esses critérios foram estabelecidos por considerar que, tendo participado do processo de tutoria e implantação, os profissionais conseguiriam perceber as mudanças no processo de trabalho após a implantação do modelo. Foram excluídos aqueles em licença de saúde ou outro tipo de afastamento no período da coleta de dados.

\section{Procedimentos}

Durante as reuniões de grupo, os pesquisadores registraram notas com as suas percepções. As reuniões de grupo foram gravadas com recursos de áudio. As gravações foram transcritas pelos pesquisadores e enviadas por correio eletrônico para que os gestores das unidades pudessem fazer a leitura $\mathrm{e}$ validação das informações juntamente com a equipe. Após a confirmação pelos gestores, procedeu-se a análise temática de conteúdo, proposta por Bardin ${ }^{17}$. Seguiu-se as seguintes etapas: a) pré-análise, onde foi realizada a organização e leitura cuidadosa de todo material; b) exploração do material, na qual buscou-se a aproximação entre as falas, análise da temática e divisão do texto por aproximação e similaridade em temas principais; e c) tratamento dos resultados: inferência e a interpretação, momento em que as categorias que foram utilizadas como unidades de análise foram analisadas à luz da literatura atual.

Os participantes foram identificados pela letra "P", que se refere a profissional, seguida das letras "AP", se vinculado à Atenção Primária, ou "AE", se vinculado à Atenção Especializada. E, 
posteriormente, o número arábico correspondente à sequência das falas para preservar a identidade dos participantes.

$\mathrm{O}$ estudo atendeu aos preceitos éticos estabelecidos na Resolução $n^{\circ}$ 466/2012 e foi aprovado pelo Comitê de Ética em Pesquisa da Pontifícia Universidade Católica do Paraná, sob o parecer $\mathrm{n}^{\mathrm{o}}$ 2.424.071/2017 e suas respectivas instituições coparticipantes. Seguiram-se as Diretrizes de Critérios Consolidados para Relatos de Pesquisa Qualitativa (COREQ).

\section{Resultados}

Para avaliar a implantação do Modelo de Atenção às Condições Crônicas foram considerados os depoimentos dos profissionais de saúde norteados pelo instrumento IEMAC-ARCHO 36, composto por seis dimensões do MACC, sendo elas: organização do sistema de saúde; saúde compartilhada; modelo assistencial; autocuidado; apoio à tomada de decisão; e sistema de informação. As categorias de análise foram estruturadas a partir de cada dimensão.

\section{Dimensão 1: organização do sistema de saúde}

A liderança como alicerce para implantação do MACC

Foi evidenciada, nessa dimensão do modelo, a importância das lideranças regionais e estadual em ofertar recursos e dar subsídios à implantação do modelo. As equipes destacaram que a iniciativa de implantação surgiu por meio da Secretaria de Saúde Estadual, que ofertou recursos humanos para capacitação da equipe. E houve um esforço das lideranças regionais em estruturação física.

$\mathrm{Eu}$ acredito que só está sendo possível implantar o MACC porque o secretário está viabilizando os recursos, as tutorias, as capacitações
[...] claro, com a ajuda da SESA. (PAP1).

Houve todo um investimento para reformar o prédio do $\mathrm{C}^{*}$ para acolher melhor a demanda dos pacientes do MACC e isso só foi possível, pois os gestores municipais se reuniram e fizeram o repasse para isso. (PAE1). [*identidade do serviço preservada].

Os indicadores de saúde como ferramenta gerencial

Ambos os serviços identificaram limitações na definição de indicadores de resultado.

Estamos registrando em planilhas o que o tutor solicitou, mas não está bem claro como vamos usar a informação. (PAP2).

Criamos várias planilhas, estamos anotando tudo que achamos importante, depois mandamos os relatórios para SESA ver. (PAE2.)

De fato criamos as planilhas dos indicadores, mas só fazemos o registro, não fazemos reunião para discutir e analisar. (PAE3).

Gastamos muito tempo alimentando as planilhas, pois só o enfermeiro pode fazer. No período que a $\mathrm{F}^{*}$ esteve de férias, ficou sem registrar, pois eu não dava conta de alimentar a minha planilha e a dela. (PAE2). [*identidade do profissional preservada].

\section{Dimensão 2: saúde compartilhada}

A intersetorialidade como um campo a ser explorado

As equipes dos serviços de saúde demonstraram desconhecer a estratégia de mapa de recursos comunitários propostos pelo instrumento e apenas a AAE reconheceu fazer uso dos recursos disponíveis.

Esse mapa dos recursos comunitários nós não temos, sei que tem a 
academia ao ar livre, o NASF né? (PAP3).

Mapa de recursos nós não construímos, mas meio que sabemos os fluxos de encaminhamento. (PAE1).

Sempre que necessário nós encaminhamos para os outros pontos da rede, para hospital e até mesmo para unidade básica, mas esse mapa desenhado não existe. (PAE3).

\section{Dimensão 3: Modelo assistencial}

\section{A compreensão do Modelo como estratégia ao cuidado crônico}

Essa dimensão do instrumento possui dezessete perguntas a respeito do MACC e gerou diversas discussões entre as equipes. A AAE destacou positivamente a implantação de plano de ação, integração para autocuidado, plano terapêutico e administração segura e revisada dos medicamentos.

Várias coisas a gente mudou com o MACC, agora todos os pacientes recebem prescrição para autocuidado de todos os profissionais que realizam consulta [...] a gente anexa o prontuário MACC e a unidade de saúde pode continuar acompanhando. (PAE4).

O paciente MACC de alto risco vem para cá e passa por consulta com todos os profissionais, médico, enfermeiro, farmacêutico, nutricionista. E todo mundo registra o plano de cuidado. (PAE5).

Os profissionais da APS destacaram positivamente a conciliação farmacológica, assistência emergencial ao doente crônico e a relação dos processos interconsultas.

Por conta da tutoria, a gente começou a rever a medicação do idoso e tentar desmedicalizar a polifarmácia. (PAP4).

A gente reorganizou, agora não tem mais hospital, mas a unidade serve de pronto atendimento. Sempre que temos alguma emergência aqui na unidade, o paciente é encaminhado para lá. Tem mais estrutura, recebe uma assistência melhor. (PAP5).

A gente sempre anota na planilha quando é a data da próxima consulta ou, também, quando tem consulta no ambulatório, para monitorar o retorno né? (PAP6).

A distância entre o que se diz e o que se faz

Os profissionais da APS apresentavam-se mais rigorosos em suas avaliações. E decidiram que as atividades realizadas que não tinham registros em prontuário ou planilhas não seriam pontuadas no instrumento como executadas ou atingidas.

A gente faz o monitoramento do plano de cuidados, mas hoje não temos como provar, pois a Agente Comunitária de Saúde (ACS) faz e não temos registro em planilha. Então, se não tem como provar, nós não podemos dizer que fazemos. (PAP4).

Uma falha muito grande é não ter esses alertas no prontuário para avisar quando o controle do paciente não está adequado. Essa informação é importante, talvez devêssemos aplicar né? (PAP3).

\section{Dimensão 4: Autocuidado}

\section{Do modelo prescritivo ao modelo e apoio}

Na dimensão de autocuidado, os profissionais da AAE se mostraram bastante otimistas e convictos de atender as propostas do modelo. A APS novamente discutiu sobre várias ações que desenvolvem, mas que não registram.

Todas essas coisas aí a gente faz [...] fazemos educação terapêutica, desenvolvemos as habilidades para autocuidado, fazemos grupos. (PAE2). 
O autocuidado é assim: o paciente passa na consulta, o profissional orienta o que ele precisa fazer e registra no prontuário. Daí depois tem que monitorar né? Para ver se ele faz direitinho. (PAE1).

Depois de o paciente passar por todos os profissionais aqui, nós imprimimos a folha com as orientações, o paciente leva para casa, esse é o plano. Daí a APS que precisa monitorar né? (PAE5).

Nós realizamos grupos, prescrevemos o plano de cuidados, mas tem muitos pacientes que não seguem. (PAP1).

A ACS vai nas casas, pede pelo plano de cuidados, pergunta se eles estão fazendo, mas tem paciente que não cuida, não faz dieta. (PAP5).

\section{Dimensão 5: Apoio à tomada de decisão}

\section{Tutoria como apoio à tomada de decisão}

Os profissionais da AAE e APS identificam o suporte da tutoria/consultoria do CONASS como um apoio à tomada de decisão. Entretanto, o matriciamento é pouco explorado como estratégia de discussão de casos clínicos e capacitação dos profissionais especialistas para os profissionais da APS.

O doutor M.A* veio aqui, ensinou a gente como fazer, ele apresentou o instrumento IVCF-20. (PAE6). [*identidade do profissional preservada].

Sempre que temos qualquer dúvida, a gente manda e-mail para SESA, eles sempre dão suporte [...] tem os consultores né? O professor E.* veio ele mesmo ensinar a gente. (PAE7). [*identidade do profissional preservada].

Não enxergo que utilizamos protocolos clínicos compartilhados, acho que ainda não estamos bem alinhados com a AAE. A gente segue o que os consultores do CONASS mandam fazer né? Mas conversamos pouco com a AAE, isso falta. (PAP10).

Algoritmos de alerta como um desafio a ser superado

Os profissionais da APS identificam que essa dimensão não está bem desenvolvida, pois carece de protocolos clínicos com algoritmos de suporte à intervenção terapêutica com base nos protocolos clínicos e diretrizes incorporados ao prontuário eletrônico.

Realmente não temos esse algoritmo, o prontuário eletrônico não faz isso. Seria muito bom, facilitaria muito? (PAP11).

Tem as linhas guia, mas as diretrizes terapêuticas tem que ficar pesquisando cada vez que quer tirar uma dúvida. O sistema não informa essas coisas não. Seria bom se ele deixasse de cor diferente conforme o risco estratificado. (PAP1).

\section{Dimensão 6: sistemas de informação}

Sistema de informação: a dicotomia da tecnologia de informação - um sistema de apoio ou de restrições?

Com relação à dimensão do sistema de informação, a AAE considera bem desenvolvida pelo fato de ter sistema de prontuário eletrônico. A APS foi novamente mais crítica e identificou falhas no sistema.

É ótimo né? O prontuário é eletrônico. (PAE7).

O que achamos muito ruim é que não conseguimos acessar o que os profissionais do ambulatório fazem, sempre depende de o paciente trazer de volta o prontuário físico, [...] mas estamos tentando integrar os sistemas agora. (PAP7).

O que incomoda é o que o sistema não gera relatórios, gastamos muito tempo alimentando informações em planilhas. (PAP8). 
autocuidado como um modelo prescritivo, que deve ser elaborado pelo profissional de saúde e executado pelo paciente. $\mathrm{Na}$ literatura, encontram-se disponíveis materiais de apoio aos profissionais de saúde com orientações sobre o autocuidado apoiado, que podem contribuir para a qualificação dos profisssionais. A literatura esclarece que o autocuidado apoiado deve ser construído coletivamente entre profissional de saúde e paciente, traçando metas, estratégias de cuidado, empoderamento, letramento em saúde e monitoramento das ações ${ }^{21}$.

O MACC caracteriza o autocuidado como objeto para o empoderamento das pessoas, para que possam cuidar da sua saúde, de modo a reconhecer o papel do usuário no gerenciamento da sua saúde e desenvolvimento de um senso de autorresponsabilidade ${ }^{9}$. Estratégias, como fixação de metas, elaboração de planos de cuidado, tecnologias de solução de problemas e gerenciamento e uso de programas de apoio, podem contribuir para atingir a dimensão.

$\mathrm{Na}$ dimensão "Apoio a tomada de decisão clínica", os resultados evidenciaram a presença de Linhas Guias para o Cuidado Crônico, mas não rotineiramente utilizadas pelos profissionais. Destaca-se a importância de ações de educação permanente aos profissionais de saúde para que eles estejam sempre atualizados com as novas evidências, o que exige métodos educacionais que permitam mudar os comportamentos dos profissionais ${ }^{22}$. As decisões clínicas devem ser tomadas com base em diretrizes clínicas construídas a partir de evidências científicas. Essas diretrizes clínicas devem ser discutidas em um diálogo com os usuários, de forma que possam compreender da melhor forma a atenção à saúde prestada ${ }^{23}$.

Observa-se que os profissionais ainda são "tutores dependentes", não assumindo o papel de Gerenciadores de caso e contando com a definição e contribuição dos profissionais tutores do
CONASS. O MACC preconiza que a definição de papéis e distribuição de tarefas entre os membros da equipe multiprofissional de saúde deve estar clara, além da introdução de novas formas de atenção, como a atenção compartilhada em grupo, atenção à distância e a atenção contínua. Preconiza, também, que haja o monitoramento regular das pessoas que vivem com condições crônicas pela equipe de saúde, para que os usuários não fiquem desassistidos ${ }^{9}$. Desse modo, propõe-se a definição de "Gerenciadores de caso", profissionais que coordenam esse processo de transição entre os níveis de atenção, mantendo contato para que não haja o abandono do usuário e perda da continuidade do cuidado.

A dimensão "Sistemas de informação" apresentou-se dicotômica na opinião dos profissionais da APS em relação aos profissionais da AAE. É possível que a má interpretação das funcionalidades do sistema de informação mascarem o entendimento de instruções ou recursos do modelo e que algumas equipes não tenham completo entendimento do processo de melhora até que estejam nele $^{24}$. Observa-se que existem avanços necessários para uma melhor aplicabilidade e comunicação por meios dos sistemas de saúde, como, por exemplo, a integração do prontuário eletrônico. $\mathrm{O}$ estudo realizado em Santo Antônio do Monte também evidenciou a limitação dos sistemas de informação, bem como de acesso aos recursos de internet ${ }^{18}$.

O MACC preconiza que haja uma utilização rotineira de prontuários clínicos informatizados, com provimento de alertas, lembretes e de feedbacks oportunos para os profissionais de saúde e para as pessoas usuárias. Concomitantemente, que haja a identificação de subpopulações relevantes, em função de riscos e elaboração de um plano de cuidado individual para cada usuário e monitoramento do desempenho da equipe de saúde e do sistema de atenção à saúde ${ }^{9}$. 
Como fragilidades apontadas, a literatura afirma que a falta de treinamentos e capacitações para utilização dos Sistemas de Informação, a lentidão na incorporação de novas tecnologias e a utilização de múltiplos sistemas de informação podem dificultar na filtragem do conhecimento desejado, do preenchimento e da utilização pelo grande volume de dados a serem preenchidos ${ }^{24}$. E afirma que a premissa básica de um sistema de informações em saúde é subsidiar informações necessárias para que os profissionais de saúde possam desempenhar suas atribuições com eficiência e eficácia, de modo a contribuir para a melhoria da qualidade da saúde da população ${ }^{19}$.

\section{Conclusão}

A percepção dos profissionais de saúde com relação à implantação do Modelo de Atenção às Condições Crônicas permitiu identificar e compreender as seis dimensões do MACC e evidenciou que apesar de apresentar avanços e mudanças no processo de cuidado crônico, demonstrou limitações e ritmo lento para alcançar mudança transformacional, especialmente, atrelada aos indicadores de saúde, mapa de ações interprofissionais, plano de autocuidado apoiado e sistemas de informação.

$O$ processo de autoavaliação por meio do IEMAC-ARCHO 36 pelas equipes de saúde oferece a oportunidade para identificação de pontos fortes e áreas de melhoria para avançar na mudança de modelo da atenção às condições crônicas, além de oportunizar uma reflexão entre a equipe de profissionais sobre $\mathrm{o}$ atendimento de seus pacientes crônicos e de compartilhar informações, experiências e perspectivas. Pode, também, ser utilizado como roteiro por tomadores de decisão, gestores e líderes. Sugere-se que a aplicação do instrumento, por meio de reuniões de equipe, torne-se uma ferramenta gerencial rotineira com avaliações semestrais ou anuais para acompanhamento da evolução da implantação do modelo.

Como limitações do estudo, devese levar em conta que o instrumento IEMAC-ARCHO 36 auxilia a reflexão e percepção dos profissionais de saúde sobre o modelo e o atendimento prestado ao paciente crônico. Tal como acontece com outros instrumentos de autoavaliação, essas percepções podem ser influenciadas por motivações, expectativas e pela própria compreensão e interpretação das intervenções pelos respondentes.

$\mathrm{O}$ ineditismo do uso do instrumento para conhecer a percepção dos profissionais de saúde no Brasil com relação à implantação do MACC traz como benefícios a inovação de novas ferramentas para avaliação. E contribui para a tomada de decisões dos gestores e para identificar os aspectos que podem ser otimizados para o pleno funcionamento do modelo. 


\section{Referências}

1. Ministério da Saúde (BR). Diretrizes para o cuidado das pessoas com doenças crônicas nas redes de atenção à saúde e nas linhas de cuidado prioritárias. Brasília: Ministério da Saúde. 2013[citado em 2020 set. 1]. Disponível em: https://bvsms.saude.gov.br/bvs/publicacoes/diretrizes\%20_cuidado_pessoas\%20_doencas _cronicas.pdf

2. World Health Organization. Noncommunicable diseases progress monitor 2020. Geneva: World Health Organization. 2020 [citado em 2020 ago. 8]. Disponível em: https://www.who.int/publications/i/item/ncd-progress-monitor-2020

3. World Health Organization. Time to Delivery. Report of the WHO Independent HighLevel Commission on Noncommunicable Diseases. Geneva: WHO. 2018 [citado em 2020 mai. 07]. Disponível em: https://apps.who.int/iris/bitstream/handle/10665/272710/9789241514163-eng.pdf

4. Malta DC, França E, Abreu DMX, Perillo RD, Salmen MC, Teixeira RA, et al. Mortalidade por doenças não transmissíveis no Brasil, 1990 a 2015, segundo estimativas do estudo de Carga Global de Doenças. São Paulo Med J. 2017;135(3):213-21. Disponível em: http://www.scielo.br/scielo.php?script=sci_arttext\&pid=S1415790X2015000600003\&lng=en

5. Malta DC, Stopa SR, Szwarcwald CL, Gomes NL, Silva Júnior J B, Reis AAC dos. A vigilância e o monitoramento das principais doenças crônicas não transmissíveis no Brasil - Pesquisa Nacional de Saúde, 2013. Rev. bras. Epidemiol. 2015[citado em 2020 abr.14]; 18(Suppl 2):3-16. Disponível em: https://www.scielo.br/pdf/rbepid/v18s2/19805497-rbepid-18-s2-00003.pdf

6. Ministério da Saúde (BR). Vigitel Brasil 2019: vigilância de fatores de risco e proteção para doenças crônicas por inquérito telefônico: estimativas sobre frequência e distribuição sociodemográfica de fatores de risco e proteção para doenças crônicas nas capitais dos 26 estados brasileiros e no Distrito Federal em 2019. Brasília: Ministério da Saúde. 2020 [citado em 2020 set. 15]. Disponível em: http://bvsms.saude.gov.br/bvs/publicacoes/vigitel_brasil_2019_vigilancia_fatores_risco.p $\mathrm{df}$

7. World Health Organization. Prevention and control of non-communicable diseases: Formal meeting of Member States to conclude the work on the comprehensive global monitoring framework, including indicators, and a set of voluntary global targets for the prevention and control of non-communicable diseases. Report by the Director-General. 2012 [citado em 2020 mai. 07]. Disponível em: http://apps.who.int/gb/ebwha/pdf_files/EB132/B132_6-en.pdf

8. Ministério da saúde (BR). Plano de ações estratégicas para o enfrentamento das doenças crônicas não transmissíveis (DCTN) no Brasil 2011-2022. Brasília: Ministério da Saúde. 2011 [citado 2020 abr. 26]. Disponível em: http://bvsms.saude.gov.br/bvs/publicacoes/plano_acoes_enfrent_dent_2011.pdf 
9. Mendes EV. Desafios do SUS. Brasília, DF: CONASS. 2019 [citado em 2020 mai. 12]. Disponível em: https://www.conass.org.br/biblioteca/desafios-do-sus/

10. Wagner EH. Organizing Care for Patients With Chronic Illness Revisited. Milbank Q. 2019 [citado em 2020 abr. 12]; 97(3): 659-64. Disponível em: https://pubmed.ncbi.nlm.nih.gov/31424130

11. Dahlgren G, Whitehead M. European strategies for tackling social inequities in health: Levelling up Part 2. Copenhage. 2007 [citado 2020 jun. 14]. Disponível em: http://www.euro.who.int/_data/assets/pdf_file/0018/103824/E89384.pdf

12. Porter M, Kellogg M. Kaiser Permanente: an integrated health care experience. Rev Innovación Sanit y Atención Integr. 2008 [citado em 2020 jun. 26];1(1):1-8. Disponível em:

https://www.sefap.org/media/upload/arxius/formacion/aula_fap_2010/bibliografia/Kaiser 2009.pdf

13. Evangelista MJ de O, Guimarães AM D’ÀN, Dourado EMR, Vale FLB do, Lins MZS, Matos MAB de et al . O Planejamento e a construção das Redes de Atenção à Saúde no DF, Brasil. Ciênc. saúde coletiva. 2019 [citado em 2020 mai. 16]; 24(6): 2115-2124. Disponível em: https://www.scielo.br/pdf/csc/v24n6/1413-8123-csc-24-06-2115.pdf

14. Dalcuche MG, Mendes EV. A integração em Rede da Atenção Primária (APS) com a Atenção Ambulatorial Especializada (AAE) no estado do Paraná. Espaço para a saúde. 2017[citado em 2020 jun. 16]; 18 (1): 31-37. Disponível em: http://espacoparasaude.fpp.edu.br/index.php/espacosaude/article/view/341

15. Nuño R, Fernández P, Mira JJ, Toro N, Contel JC, Guilabert M, Solas O. Desarrollo de IEMAC, un Instrumento para la Evaluación de Modelos Asistenciales ante la Cronicidad. Gac Sanit. 2013 [citado em 2020 abr. 14]; 27 (2): 128-34. Disponível em: https://www.sciencedirect.com/science/article/pii/S0213911112002166

16. Santos ZFDG, Werneck RI. Avaliação de serviços de saúde como indutor do modelo de atenção e disponibilização de instrumento de autoavaliação para serviços de cuidado de condições crônicas IEMAC-ARCHO validado. [tese]. Curitiba: Pontifícia Universidade Católica do Paraná; 2020.

17. Bardin L. Análise de conteúdo. 1ª ed. São Paulo: Edições 70; 2016.

18. Andrade MV, Noronha K, Cardoso CS, Oliveira CDL, Calazans JA, Souza MN. Challenges and lessons from a primary care intervention in a Brazilian municipality. Rev. Saúde Pública. 2019 [citado em 2020 ago. 09] ; 53: 45.Disponível em: http://www.scielo.br/scielo.php?script=sci_arttext\&pid=S0034$89102019000100240 \& \operatorname{lng}=$ en.

19. Pereira $\mathrm{B}$ dos $\mathrm{S}$, Tomasi $\mathrm{E}$. Instrumento de apoio à gestão regional de saúde para monitoramento de indicadores de saúde. Epidemiol. Serv. Saúde. 2016 Jun. [citado em 2020 ago.09]; 25(2):411-418.Disponível em:

http://www.scielo.br/scielo.php?script=sci_arttext\&pid=S2237-

96222016000200411\&lng=en. 
20. Monnerat GL, Souza RG. Intersetorialidade e políticas sociais: um diálogo coma literatura atual. In: Monnerat GL, Almeida NLT, Souza RG. (orgs.) A intersetorialidade na agenda das políticas sociais. Campinas: Papel Social; 2014; 41-54.

21. Cavalcanti AM, Oliveira ACL. (Org.). Autocuidado apoiado: manual do profissional de saúde. Curitiba: Secretaria Municipal da Saúde; 2012.

22. Silva LAA da, Soder RM, Petry L, Oliveira IC. Educação permanente em saúde na atenção básica: percepção dos gestores municipais de saúde. Rev. Gaúcha Enferm. [Internet]. 2017 [citado em 2020 jul.17]; 38(1): e58779. Disponível em: https://www.scielo.br/pdf/rgenf/v38n1/0102-6933-rgenf-1983-144720170158779.pdf

23. Cruz A. Inovação na Atenção Especializada no Brasil. Rev Consensus [internet]. 2015[citado em: 2020 mai. 28]; 16,14-22. Disponível em: https://www.conass.org.br/biblioteca/pdf/revistaconsensus_16.pdf.

24. Pinheiro ALS, Andrade KTS, Silva D de O, Zacharias FCM, Gomide MFS, Pinto IC. Health management: the use of information systems and knowledge sharing for the decision making process. Texto contexto - enferm. 2016 [citado em 2020 ago. 20]; 25(3): e3440015. Disponível em: https://www.scielo.br/pdf/tce/v25n3/0104-0707-tce-25-033440015.pdf.

\section{Como citar este artigo:}

Theis LC, Mikosz DM, Rosa SV, Moysés ST, Moraes TP. Percepção dos profissionais de saúde em relação à implantação do Modelo de Atenção às Condições Crônicas. Rev. Aten. Saúde. 2021; 19(68): 7-20. 